\title{
UNE FORMULE DE HADAMARD DANS DES PROBLEMES
}

\section{D'OPTIMAL DESIGN}

\author{
A. Dervieux \\ B. Palmerio \\ Université de Nice \\ Institut de Mathématiques et Sciences Physiques \\ Parc Valrose \\ 06034 NICE Cedex - France
}

Nous nous intéressons à des problèmes d'optimal design qui peuvent être formulés ainsi

Problème : Minimiser la fonctionnelle coût $j(\Omega)$ pour $\Omega$ appartenant à $\psi_{\text {ad }}$; où $u_{\text {ad }}$ est une famille de domaines $\Omega$ de $\mathbb{R}^{\mathrm{n}}$.

Pour résoudre numériquement un tel problème une direction d'approche naturelle consiste à chercher la dérivêe de la fonctionnelle $\mathrm{j}$ par rapport au domaine $\Omega$. Hadamard [9] en 1908 calcula la dérivée de la plus petite valeur propre de certains opérateurs par rapport au domaine géométrique.

Nous étudions ici l'optimal design de systèmes régis par des problèmes aux limites elliptiques bien posés $\left({ }^{1}\right)$. En particulier nous nous referrerons au long de cet article au problème modèle suivant :

Problème modèle : Minimiser dans un ensemble $u_{\text {ad }}$ la fonctionnelle

$$
j(\Omega)=\frac{1}{2}\left\|z(\Omega)-z_{d}\right\|^{2}{ }^{2}\left(\Omega_{0}\right)
$$

où $z(\Omega)$ est la solution de l'équation d'état dans $\Omega$

$$
\left\{\begin{array}{l}
z(\Omega) \in \mathrm{H}^{1}(\Omega) \text { et } \forall \varphi \in \mathrm{H}^{1}(\Omega) \\
\int_{\Omega}\{\nabla \mathrm{z}(\Omega) \cdot \nabla \varphi+\mathrm{z}(\Omega) \varphi-f \varphi\} d v=0
\end{array}\right.
$$

et $u_{\text {ad }}$ est un ensemble d'ouverts vérifiant

$$
\forall \Omega \in u_{\text {ad }}, \quad \Omega_{0} \subset \Omega \subset B
$$

(1) Pour des problèmes aux valeurs propres, le lecteur pourra se réferrer à [12] et [7] et à la bibliographie de ces travaux. 
$\Omega_{0}$ et $B$ êtant deux ouverts fixes de $\mathbb{R}^{n}$ et

$$
z_{d} \in L^{2}\left(\AA_{0}\right): f \in L^{2}(B)
$$

Le plan que nous suivrons est le suivant

1 Formulation du problème

1. 1 Repérage du domaine

1.2 Notations

1.3 Calcul formel du gradient

2 Transport sur un ouvert fixe : dérivabilité

3 Prolongement à un ouvert fixe : formule de Hadamard

4 Exemples

5 Conclusion 


\section{Formulation du Problème}

\subsection{Repérage du domaine}

Nous utiliserons un repérage classique pour ce genre de problèmes (cf. [3] ) ; soit $\mathrm{k}$ un entier supérieur ou égal à $2, \quad B$ un ouvert borné de $\mathbb{R}^{\mathrm{n}}$ on pose $\vartheta_{k}=\{T$ difféomorphisme de $B$ dans $B$

Soit $\Omega$ un ouvert fixe de fermeture incluse dans B, de classe $C^{k}$ (cf. par exemple [11] ) ; on s'intéresse aux domaines images de $\Omega$ par certains éléments de of : plus précisément l'ensemble $U_{\text {ad }}$ des domaines admissibles est de la forme (1.2) $\quad u_{\mathrm{ad}}=\left\{\Omega_{\mathrm{T}}=\mathrm{T}(\Omega) ; \mathrm{T} \in \Omega_{\mathrm{ad}}\right\}$ où $g_{\mathrm{ad}}$ désigne l'ensemble des difféomorphismes admissibles $d_{\mathrm{ad}} \subset d_{\mathrm{k}}$.

\subsection{Notations}

Il sera plus pratique désormais de considérer le difféomorphisme comme nouveau contrôle et de formuler le problème général de la façon suivante :

(P) Minimiser dans $\vartheta_{\text {ad }}$ la fonctionnelle

$$
\text { (1.3) } \quad j(T)=J(T, z(T))
$$

où $z(T)$ est la solution d'un problème aux limites dans $\Omega_{T}$ noté ainsi

$$
\text { (1.4) }\left\{\begin{array}{l}
z(T) \in Z_{T} \\
\Phi(T, z(T))=0 \text { dans } Q_{T}^{\prime}
\end{array}\right.
$$

et avec

$$
\text { (1.5) }\left\{\begin{array}{l}
\mathrm{J}:{ }^{\vartheta_{\mathrm{ad}}} \times \mathrm{Z}_{\mathrm{T}} \rightarrow \mathbb{R} \\
\mathbf{T}:{ }_{\mathrm{ad}} \times \mathrm{Z}_{\mathrm{T}} \rightarrow \mathbf{Q}_{\mathrm{T}}^{\prime}
\end{array} ;\right.
$$

$\mathrm{Z}_{\mathrm{T}}$ et $\mathbf{Q}_{\mathrm{T}}^{\prime}$ sont des espaces de Banach dépendant du contrôle $\mathrm{T}: \mathrm{J}$ et $\Phi$ ne sont pas des applications.

Cas du problème modèle: On pose pour $\mathrm{T}$ appartenant à $\theta_{\mathrm{ad}}$

$$
\begin{aligned}
& \mathrm{Z}_{\mathrm{T}}=\mathrm{H}^{1}\left(\Omega_{\mathrm{T}}\right) \quad \mathrm{Q}_{\mathrm{T}}=\mathrm{H}^{1}\left(\Omega_{\mathrm{T}}\right) \\
& \forall \varphi \in \mathrm{Z}_{\mathrm{T}}, \Psi \in \mathrm{Q}_{\mathrm{T}}
\end{aligned}
$$


(1.6)

$$
\begin{aligned}
& J(T, \varphi)=\frac{1}{2}\left\|\varphi-z_{d}\right\|^{2}{ }^{2}\left(\Omega_{0}\right) \\
& {[\Phi(T, \varphi), \Psi]_{Q_{T}^{\prime} \times Q_{T}}=} \\
& \left.\quad \int_{\Omega_{T}}\{<\nabla \varphi, \nabla \Psi\rangle+\varphi \Psi-f \Psi\right\} d v
\end{aligned}
$$

Le symbole $<,>$ désigne, sauf mention contraire, le produit scalaire de $\mathbf{R}^{\mathrm{n}}$.

\section{3 Calcul formel du gradient}

En dêrivant formellement l'équation (1.4) on obtient

$$
\frac{\partial \Phi}{\partial T}+\frac{\partial \Phi}{\partial \varphi} \cdot \frac{d z}{d T}(T)=0
$$

introduisons l'état adjoint suivant

$$
\begin{aligned}
& \mathrm{p}(\mathrm{T}) \in \mathrm{Q}_{\mathrm{T}} \quad \text { et } \forall \varphi \in \mathrm{Z}_{\mathrm{T}} \\
& {\left[\frac{\partial \Phi}{\partial \varphi}(\mathrm{T}, \mathrm{z}(\mathrm{T})) \cdot \varphi, \mathrm{p}(\mathrm{T})\right]=\frac{\partial J}{\partial \varphi}(\mathrm{T}, \mathrm{z}(\mathrm{T})) \cdot \varphi}
\end{aligned}
$$

et utilisons la dérivation composée

$$
\frac{d j}{d T}(T)=\frac{\partial J}{\partial T}(T, z(T))+\frac{\partial J}{\partial \varphi}(T, z(T)) \cdot \frac{d z}{d T}(T)
$$

nous obtenons

$$
\frac{d j}{d T}(T)=\frac{\partial J}{\partial T}(T, z(T))-\left[\frac{\partial \Phi}{\partial T}(T, z(T)), p(T)\right] \text {. }
$$

Le but de cet article est de présenter un contexte général où ce calcul formel est justifié. Nous mettrons aussi en évidence dans quelques exemples que le deuxième membre de (1.10) est une intégrale de surface du même type que celles introduites par J.Hadamard.

Mais tout d'abord nous nous intêresserons à la dérivabilité de la fonctionnelle $j$ : c'est l'objet du paragraphe 2 .

\section{Transport sur un ouvert fixe. Dérivabilité}

Soit $\mathrm{T}$ un élément de $d_{\mathrm{ad}}, \theta$ et $s$

$$
\begin{array}{r}
\theta \in\left[\mathrm{C}^{\mathrm{k}}(\overline{\mathrm{B}})\right]^{\mathrm{n}} \\
\delta>0
\end{array}
$$

tels que

$$
T+t \theta \in d_{a d} \quad \forall t \in I=[0, \delta[;
$$

Dans ce qui suit $\mathrm{T}$ dêsignera sans ambiguité possible tantôt un élément fixe vérifiant la condition (2.1) tantôt une variable décrivant l'ensemble $\jmath_{a d}$ • Nous allons êtudier la dérivabilité au sens de Gâteaux de la fonctionnelle j dans 
la direction $\theta$. La principale difficulté du contexte précédent provient du fait que l'espace des fonctions d'état varie avec le contrôle. Une idée naturelle pour se ramener à des espaces fixes consiste à transporter l'équation d'état sur l'ouvert fixe $\Omega$ en utilisant le difféomorphisme $\mathrm{T}$; d'où l'hypothèse

(H 1) Il existe un espace de Banach $Z$ et un espace de Banach réflexif $Q$ de fonctions définies sur $\Omega$ tels que pour tout élément $T$ de $d_{\text {ad }}$ l'application $\varphi \rightarrow \varphi \circ T$ est un isomorphisme de $Z_{T}$ sur $Z\left(\right.$ resp. $Q_{T}$ sur Q).

Considérons l'êtat transporté

$$
\tilde{z}(T)=z(T) \cdot T
$$

c'est la solution du système

$$
\left\{\begin{array}{l}
\tilde{z}(T) \in \quad Z \\
\tilde{\Phi}(T, \tilde{z}(T))=0 \text { dans } Q^{\prime}
\end{array}\right.
$$

où l'application $\widetilde{\Phi}$ est définie de la manière suivante

$$
\left\{\begin{aligned}
& \tilde{\tau}: d_{\mathrm{ad}} \times \mathrm{Z} \rightarrow \mathrm{Q}^{\prime} \\
& {[\widetilde{\Phi}(\mathrm{T}, \varphi), \Psi]=\left[\Phi\left(\mathrm{T}, \varphi \circ \mathrm{T}^{-1}\right), \Psi \circ \mathrm{T}^{-1}\right] }
\end{aligned}\right.
$$

et le problème $(\mathrm{P})$ (cf. le paragraphe 1.2 équivaut à $(\widetilde{\mathbf{P}})$

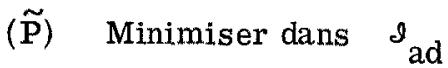

$$
j(T)=\tilde{J}(T, \tilde{z}(T))
$$

où $\tilde{\mathrm{Z}}(\mathrm{T})$ est la solution de $(2.3)$ et avec

$$
\left\{\begin{array}{l}
\tilde{J}: \quad d_{\mathrm{ad}} \times \mathrm{Z} \rightarrow \mathbf{R} \\
\tilde{J}(\mathrm{~T}, \varphi)=J\left(T, \varphi \circ \mathrm{T}^{-1}\right)
\end{array}\right.
$$

Dans le cas du problème modèle on aura

$$
\begin{aligned}
& \mathrm{Z}=\mathrm{H}^{1}(\Omega) \quad \mathrm{Q}=\mathrm{H}^{1}(\Omega) \\
& \tilde{\mathrm{J}}(T, \varphi)=\frac{1}{2}\left\|\varphi \circ T^{-1}-\mathrm{z}_{d}\right\|_{L^{2}}^{2}\left(\Omega_{0}\right) \\
& \begin{array}{l}
{[\tilde{\Phi}(\mathrm{T}, \varphi), \Psi]\left[\mathrm{H}^{1}(\Omega)\right]^{\prime} \times \mathrm{H}^{1}(\Omega)=} \\
\left.\int_{\Omega}\left\{\left\langle\mathrm{DT}^{-1} \nabla_{\varphi}, \mathrm{DT}^{-1 *} \nabla \Psi\right\rangle+\varphi \Psi-f \circ \mathrm{T}^{*}\right\}\right\}|\mathrm{DT}| \mathrm{dv}
\end{array}
\end{aligned}
$$

On voit dans cet exemple que le contrôle $T$ intervient uniquement dans les coefficients et le deuxième membre de l'équation d'êtat. Nous montrerons la dérivabilitê de $\mathrm{j}$ à partir de celle de l'application

$$
\left\{\begin{array}{l}
d_{\text {ad }} \rightarrow \mathrm{Z} \\
\mathrm{T} \rightarrow \tilde{\mathrm{Z}}(\mathrm{T})
\end{array}\right.
$$

celle-ci est une conséquence du théorème des fonctions implicites, d'où l'introduc- 
tion d'hypothèses de régularité sur les applications $\tilde{\Phi}$ et $\widetilde{J}$

(H 2) (i) L'application suivante est continue

$$
\begin{aligned}
& I \times Z \rightarrow Q^{\prime} \\
& (t, \varphi) \rightarrow \widetilde{\Phi}(T+t \theta, \varphi) .
\end{aligned}
$$

(ii) Elle est dérivable par rapport à $\varphi$ et sa dérivée partielle $\partial \widetilde{\Phi} / \partial \varphi$ est continue par rapport au couple $(t, \varphi)$.

(iii) Elle est dérivable en $(o, \tilde{z}(T))$ et l'on a

$$
\frac{\partial \widetilde{\Phi}}{\partial \varphi}(o, \widetilde{z}(T)) \in \operatorname{Isom}\left(Z, Q^{\prime}\right) \text { 。 }
$$

(H 3) Lapplication

$$
\begin{aligned}
& I \times Z \rightarrow \mathbb{R} \\
& (t, \varphi) \rightarrow \tilde{J}(T+t \theta, \varphi)
\end{aligned}
$$

est dérivable en $(0, \tilde{z}(\mathrm{~T}))$.

Proposition 2.1 - On se place sous les hypothèses (H 1) à (H 3); alors

(i) L'application (2.6) est $\mathrm{G}$-différentiable en $\mathrm{T}$ dans la direction $\theta$

$$
\widetilde{\mathrm{Z}}^{:}(\mathrm{T}, \theta)=
$$

$(2.7)$

$$
-\left[\frac{\partial \tilde{\Phi}}{\partial \varphi}(T, \tilde{z}(T))\right]^{-1} \cdot\left[\frac{d}{d t}(\tilde{\Phi}(T+t \theta, \tilde{z}(T))]_{t=0}\right.
$$

(ii) La fonctionnelle $\mathrm{j}$ est $\mathrm{G}$-différentiable en $\mathrm{T}$ dans la direction $\theta$ $j^{\prime}(T, \theta)=$

$$
\frac{d}{d t}[\tilde{J}(T+t \theta, \widetilde{z}(T))]_{t=0}-\frac{d}{d t}[\widetilde{q}(T+t \theta, \widetilde{z}(T)), \widetilde{p}(T)]_{t=0}
$$

où $\widetilde{p}(T)$ est la solution du système adjoint suivant

$$
\widetilde{\mathrm{p}}(\mathrm{T}) \in Q
$$

$$
\left[\frac{\partial \widetilde{\varphi}}{\partial \varphi}(T, \tilde{z}(T))\right]^{*} \cdot \tilde{p}(T)=\frac{\partial \widetilde{J}}{\partial \varphi}(T, \tilde{z}(T)) \text {. }
$$

Cette proposition est une adaptation du théorème des fonctions implicites ( $\mathrm{cf}$. par exemple [13] p.283). L'état adjoint est introduit comme dans [2].

\section{Application au problème modêle.}

Les hypothèses de la proposition précédente sont des hypothèses de régularités sur les données et le contrôle. Les conditions suivantes sont suffisantes pour leur vêrification

$$
\begin{aligned}
& f \in H^{1}(B) \\
& k=2 \text { dans }(2.1)
\end{aligned}
$$


L'état adjoint $\widetilde{p}(T)$ est la solution du système

$$
\begin{aligned}
& \tilde{p}(T) \in H^{1}(\Omega) \text { et } \forall \varphi \in H^{1}(\Omega) \\
& \int_{\Omega}\left\{<\mathrm{DT}^{-1 *} \nabla \tilde{\mathrm{p}}(\mathrm{T}), \mathrm{DT}^{-1} * \nabla \varphi>+\tilde{\mathrm{p}}(\mathrm{T}) \varphi\right. \\
& \left.\varphi\left(\tilde{z}(\mathrm{~T})-\mathrm{z}_{\mathrm{d}} \circ \mathrm{T}\right)\right\}|\operatorname{det} \mathrm{T}| \mathrm{dv}=0
\end{aligned}
$$

et la formule (2.8) se met sous la forme suivante

$$
j^{\prime}(T, \theta)=\int_{\Omega}\left\{\left\langle G_{0}, \theta\right\rangle \mathbb{R}^{n}+\left\langle G_{1}, D \theta{ }_{\mathbf{R}^{n^{2}}}\right\} d v\right.
$$

en posant

$$
\begin{aligned}
& \int_{\Omega}\left\langle\mathrm{G}_{0}, \theta\right\rangle \mathrm{dv}=\int_{\Omega}<\nabla \mathrm{f} \circ \mathrm{T}, \theta>\tilde{\mathrm{p}}(\mathrm{T}) \quad|\mathrm{DT}| \mathrm{dv} \\
& -\int_{\Omega_{0}}\left(\tilde{z}(T) \circ T^{-1}-z_{d}\right)<\nabla \widetilde{z}(T) \circ T^{-1}, D T^{-1} \cdot T^{-1} \cdot \theta>d v \\
& \int<\mathrm{G}_{\uparrow}, \mathrm{D} \theta>\mathrm{dv}= \\
& -\int_{\Omega}\left\{\left\langle\mathrm{DT}^{-1 *} \nabla \tilde{\mathrm{Z}}(\mathrm{T}), \mathrm{DT}^{-1} * \nabla \tilde{\mathrm{p}}(\mathrm{T})\right\rangle+\tilde{\mathrm{z}}(\mathrm{T}) \tilde{\mathrm{p}}(\mathrm{T})\right.
\end{aligned}
$$

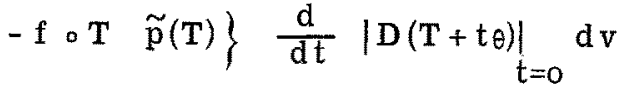

$$
\begin{aligned}
& +\int_{\Omega}\left\{<\left[D T^{-1} \cdot D \theta \cdot D T^{-1}\right]^{*} \nabla \tilde{z}(T), D T^{-1 *} \nabla \tilde{p}(T)>\right. \\
& \left.+<D T^{-I *} \nabla \tilde{z}(T),\left[D T^{-\hat{I}} \cdot D \theta \cdot D T^{-1}\right] * \nabla \tilde{p}(T)\right\}|D T| d v .
\end{aligned}
$$

Du point de vue optimisation il est utile de se placer dans un contexte hilbertien : soit $G$ une solution du problème aux limites

$$
\left\{\begin{array}{l}
\mathrm{G} \in\left[\mathrm{H}^{1}(\mathrm{~B})\right]^{\mathrm{k}} \\
\left.((\mathrm{G}, \theta)) \mathrm{H}^{1}(\Omega)\right]^{\mathrm{n}}=\int_{\Omega}\left\{\left\langle\mathrm{G}_{0}, \theta>+\left\langle\mathrm{G}_{1}, \mathrm{D} \theta\right\rangle\right\} d v ;\right.
\end{array}\right.
$$

si $G$ est suffisamment régulière, $G$ est une direction de plus grande descente pour le problème $(P)$.

Cette expression de gradient est peu commode à utiliser et nous allons mettre en évidence une expression plus simple de ce gradient.

\section{Prolongement à un ouvert fixe. Formule de Hadamard}

Comme annoncé au paragraphe 1.3 nous nous proposons d'exprimer le gradient de la fonctionnelle $j$ non plus à partir des applications $\tilde{\Phi}$ et $\tilde{J}$ mais à partir des correspondances $\Phi$ et $\mathrm{J}$ ou plus exactement à l'aide d'application $\bar{\Phi}, \overline{\mathrm{J}}$ prolongeant ces correspondances. Pour cela on introduit deux espaces de Banach $Z_{B}$ et $Q_{B}$ de fonctions définies sur $B$ vérifiant l'hypothèse

(H 5) (i) L'application

$$
\varphi \rightarrow \varphi \circ T
$$

est un automorphisme de $Z_{B}$ (resp, $\left.Q_{B}\right)$ 
ii) Il existe deux opérateurs linéaires continus et à images denses

$$
\begin{aligned}
& \mathbf{R}_{1}: \mathrm{Z}_{\mathrm{B}} \rightarrow \mathrm{Z} \\
& \mathbf{R}_{2}: \mathbf{Q}_{\mathrm{B}} \rightarrow \mathbf{Q} .
\end{aligned}
$$

De ces opêrateurs, qui seront dans les exemples des opérateurs de restriction, on dẻduit par transport

$$
\begin{aligned}
& \mathbf{R}_{1}^{\mathrm{T}}: \mathrm{Z}_{\mathrm{B}} \rightarrow \mathrm{Z}_{\mathrm{T}} \\
& \mathbf{R}_{2}^{\mathrm{T}}: \mathrm{Q}_{\mathrm{B}} \rightarrow \mathrm{Q}_{\mathrm{T}}
\end{aligned}
$$

définis par

$$
\mathbf{R}_{i}^{\mathrm{T}} \varphi=\left[\mathbf{R}_{\mathrm{i}}(\varphi \circ \mathrm{T}] \text { a } \mathrm{T}^{-1} \quad \text { pour } \mathrm{i}=1,2\right.
$$

Soient $\bar{\Phi}$ et $\bar{J}$ les prolongés de $\bar{q}$ et $J$ dans le sens suivant

$$
\begin{aligned}
& \bar{\Phi}: \mathfrak{l}_{\mathrm{ad}} \times \mathbf{Z}_{\mathrm{B}} \rightarrow \mathbf{Q}_{\mathrm{B}}^{\prime} \\
& {[\bar{\Phi}(\mathrm{T}, \varphi), \Psi]=\left[\Phi\left(\mathrm{T}, \mathbf{R}_{1}^{\mathrm{T}} \varphi\right), \mathbf{R}_{2}^{\mathrm{T}}\right]} \\
& \bar{J}: \jmath_{\mathrm{ad}} \times \mathrm{Z}_{\mathrm{B}} \rightarrow \mathbf{R} \\
& \bar{J}(\mathrm{~T}, \varphi)=\mathrm{J}\left(\mathrm{T}, \mathbf{R}_{1}^{\mathrm{T}} \varphi\right)
\end{aligned}
$$

Cas du problème modèle. On pose

et pour $i=1,2$

$$
{ }_{B}=Q_{B}=H^{1}(B)
$$

$$
\begin{aligned}
\mathrm{R}_{\mathrm{i}}: \mathrm{H}^{1}(\mathrm{~B}) & \rightarrow \mathrm{H}^{1}(\Omega) \\
\varphi & \left.\rightarrow \varphi\right|_{\Omega}
\end{aligned}
$$

alors on a

$$
\forall \varphi, \Psi \in \mathrm{H}^{1}(\mathrm{~B})
$$

(3.4) $[\bar{\Phi}(\mathrm{T}, \varphi), \Psi]=\int_{\Omega_{\mathrm{T}}}\{\langle\nabla \varphi, \nabla \Psi\rangle+\varphi \Psi-\mathrm{f} \Psi\} \mathrm{dv}$

$$
\bar{J}(T, \varphi)=\frac{1}{z}\left\|\varphi-z_{d}\right\|_{L}^{2}{ }_{L}^{2}\left(\Omega_{0}\right) \quad \text { । }
$$

Dans cet exemple, des problèmes concernant la régularité des fonctions d'état (direct et adjoint) se posent si l'on veut dériver $\tilde{\Phi}$ par rapxort à $\mathbf{T}$; dans la formulation générale ces problèmes se retrouvent dans la difficulté de déduire de la dérivabilitê de $\tilde{\Phi}(\operatorname{resp} . \tilde{J})$ celle de $\bar{\varphi}(\operatorname{resp} . \bar{J})$, difficulté qui provient du transport par le difféomorphisme (cf. les relations (2.4) et (2.5)). C'est pourquoi nous allons introduire deux espaces de fonctions plus régulières que les fonctions standard des espaces $Z_{B}$ et $Q_{B}$; les fonctions d'état devront appartenir à ces espaces; en fait, 
dans la plupart des exemples on profite d'un supplément de régularitè consécutif à la vérification de l'hypothèse (H 2) .

(H 6) Il existe deux sous espaces $Z_{r}$ et $Q_{r}$ de $Z_{B}$ et $Q_{B}$ (respectivement) tels que l'application

$$
t \rightarrow \varphi \circ(T+t \theta)
$$

soit dérivable en $\circ$ dans $Z_{B}\left(\operatorname{resp}, Q_{B}\right)$ pour tout élément $\varphi$ de $Z_{r}$ (resp. $Q_{r}$ ). Proposition 3.1 - On se place sous les hypothèses (H I) à (H 6) ; on suppose en outre que les systèmes suivants

$$
\left\{\begin{array}{l}
\bar{z}(T) \in Z_{B} \\
\bar{\Phi}(T, \bar{z}(T))=0 \text { dans } Q_{B}^{\prime}
\end{array}\right.
$$

$$
\left\{\begin{array}{l}
\overline{\mathrm{p}}(\mathrm{T}) \in \mathrm{Q}_{\mathrm{B}}, \forall \varphi \in \mathrm{Z}_{\mathrm{B}} \\
{\left[\frac{\partial \bar{\varphi}}{\partial \varphi}(\mathrm{T}, \overline{\mathrm{z}}(\mathrm{T})), \varphi, \overline{\mathrm{p}}(\mathrm{T})\right]_{\mathrm{Q}_{\mathrm{B}}^{\prime} \times \mathbf{Q}_{\mathrm{B}}}=\frac{\partial \overline{\mathrm{J}}}{\partial \varphi}(\mathrm{T}, \mathrm{z}(\mathrm{T})) \cdot \varphi}
\end{array}\right.
$$

admettent des solutions qui vêrifient les conditions de régularitê

$$
\overline{\mathrm{z}}(\mathrm{T}) \in \mathrm{Z}_{\mathrm{r}} \quad \overline{\mathrm{p}}(\mathrm{T}) \in \mathrm{Q}_{\mathbf{r}} .
$$

Alors le gradient $(2,8)$ se met sous la forme

$$
j^{\prime}(T, \theta)=\frac{d}{d t}[\bar{J}(T+t \theta, \bar{z}(T))]_{t=0}-\frac{d}{d t}[\bar{\Phi}(T+t \theta, \bar{z}(T)), \bar{p}(T)]_{t=0} .
$$

Proposition 3.2 - On se place sous les hypothèses (H 1) à (H 6) et on suppose qu'il existe un opérateur de prolongement $p$ linéaire continu de $\mathrm{Z}$ dans $\mathrm{Z}_{\mathrm{B}}$

$$
\begin{aligned}
& P: Z \rightarrow Z_{B} \\
& R_{1} \circ P=I d_{Z} ;
\end{aligned}
$$

soit $H$ un espace de Banach contenant $Z_{B}$ tel que l'application

$$
\begin{aligned}
& I \times Z_{B} \rightarrow H \\
& (t, \varphi) \rightarrow \varphi \circ[T+t \theta]^{-1}
\end{aligned}
$$

soit dérivable en $(0, \varphi)$ pour tout élément $\varphi$ de $Z_{B}$.

Alors il existe pour tout élément $T$ de $d_{\text {ad }}$ un prolongement $\bar{z}(T)$ de $z(T)$ dans $\mathrm{Z}_{\mathrm{B}}$ dans le sens suivant

$\bar{z}(T)$ est une solution de (3.7), $\quad R_{1}^{T} \bar{z}(T)=z(T)$ et tel que l'application

$$
\begin{aligned}
& \vartheta_{\text {ad }} \rightarrow \mathrm{H} \\
& \mathrm{T} \rightarrow \overline{\mathrm{z}}(\mathrm{T})
\end{aligned}
$$

admet pour Gâteaux-dérivée en $T$ dans la direction $\theta$ la solution du système 


$$
\frac{\partial \bar{\Phi}}{\partial \varphi}(T, \bar{z}(T))_{0} \bar{z}^{\prime}(T, \theta)=-\frac{d}{d \bar{t}} \bar{\Phi}(T+i \theta, \bar{z}(T))_{t=0} .
$$

\section{Remarque 3.1}

Le calcul formel de dérivation composée de paragraphe 1.3 peut être entièrement justifié via la proposition 3.2 mais sous des hypothèses plus fortes que celles de la proposition 3.1 qui n'est donc pas une conséquence de la proposition 3.2 .

\section{$4 \quad$ Exemples}

\subsection{Quelques lemmes techniques}

Nous verrons plus loin que les calculs de gradients relatifs à un certain nombre de problèmes se ramènent grâce à la proposition 3.1 à l'utilisation de quelques formules de dérivation connues en Mécanique des Milieux continus (cf. par exemple [8]).

On conserve les notations des paragraphes précédents, et on pose

$$
\mathrm{V}=\theta \cdot \mathrm{T}^{-1}
$$

et pour $t$ appartenant à l'intervalle I

$$
\Omega_{t}=(T+t \theta) \Omega
$$

On peut énoncer les lemmes suivants

Lemme 4.1 Soit $u$ un élêment de $W^{1,1}\left(\mathbb{R}^{2}\right)\left({ }^{1}\right)$ alors

$$
\left.\frac{d}{d t} \int_{\Omega_{t}} u d v=\int_{\partial \Omega_{t}} u<\vec{n}, v\right\rangle d \sigma
$$

où $\overrightarrow{\mathbf{n}}$ dêsigne un vecteur unitaire normal à $\partial \Omega_{\mathrm{t}}$ et dirigé vers l'extérieur de $\Omega_{\mathrm{t}}$.

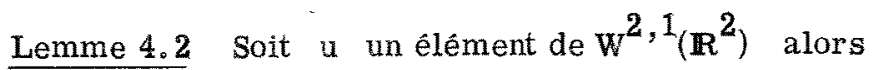

$$
\frac{d}{d t} \int_{\partial \Omega_{t}} u d \sigma=\int_{\partial \Omega_{t}}[\langle\nabla u, v\rangle+u\langle D V \cdot \vec{\tau}, \vec{\tau}\rangle] d \sigma
$$

où $\vec{\tau}$ est un vecteur tangent à $\partial \Omega_{t}$.

Remarque 4.1 - La formule précédente peut se mettre sous une forme plus simple en faisant intervenir la courbure de la frontière $\partial \Omega_{t}(c f .[11])$.

Lemme 4.3 Soient $u$ un êlểment de $\mathrm{H}^{3}\left(\mathbf{R}^{\mathrm{n}}\right)\left(^{2}\right)$, et $\mathrm{w}$ un élément de $\mathrm{H}^{2}\left(\mathbf{R}^{\mathrm{n}}\right)$ alors

$$
\frac{d}{d t} \int_{\partial n_{t}} u \quad \frac{\partial v}{\partial n} d \sigma=\int_{\partial \Omega_{t}}\{\langle\nabla u, \nabla v>+u \Delta v\}\langle\vec{n}, v\rangle \quad d \sigma
$$

Le lemme 4.3 est une conséquence de la formule de Green et du lemme 4.1.

$$
\begin{aligned}
& { }_{(1)}^{1} \mathrm{w}^{\mathrm{m}}, \mathrm{P}(\Omega)=\left\{\mathrm{u} \in \mathrm{L}^{\mathrm{P}}(\Omega) ; \forall \alpha \in \mathbb{N}^{\mathrm{n}},|\alpha| \leq \mathrm{m} \quad \mathrm{D}^{\alpha} \mathrm{u} \in \mathrm{L}^{\mathrm{P}}(\Omega)\right\} \quad \forall \Omega \subset \mathbb{R}^{\mathrm{n}} . \\
& \left({ }^{2}\right) \mathrm{H}^{\mathrm{S}}(\Omega)=\mathrm{W}^{\mathrm{s}, 2}(\Omega)
\end{aligned}
$$




\subsection{Résultats}

Pour plus de simplicitê on se bornera à définir pour chaque exemple une application $\bar{\Phi}$ vérifiant les hypothèses de la proposition 3.1 ainsi que les hypothèses de régularitê requises pour les données et pour 1'ouvert; dans la plupart des cas ces hypothèses sont plus que suffisantes pour garantir l'existence des expressions obtenues; on peut envisager d'améliorer ces hypothèses par un raisonnement par densité (cf. [4] ). On montre par ailleurs (cf. [5 ] ) l'existence de formules a nalogues (intêgrales sur le bord variable) sous des hypothèses beaucoup moins restrictives.

Exemple $n^{\circ} 1$ (Problème modèle) -

On suppose que

et

$$
a_{\mathrm{ad}} \subset d_{2} \quad(\operatorname{cf} \cdot(1.1))
$$

on introduit l'état adjoint suivant

$$
\left\{\begin{array}{l}
-\Delta p+p=x_{\Omega_{0}}\left(z-z_{d}\right) \text { dans } \Omega_{T}\left({ }^{1}\right) \\
\frac{\partial p}{\partial n}=0 \quad \text { sur } \quad \partial \Omega_{T}
\end{array}\right.
$$

et le gradient est obtenu sous la forme suivante

$$
\left.\mathbf{j}^{\prime}(\mathrm{T}, \theta)=-\int_{\partial \Omega_{\mathrm{T}}}\{\langle\nabla \mathrm{z}, \nabla \mathrm{p}\rangle+\mathrm{zp}-\mathrm{fp}\}<\overrightarrow{\mathrm{n}}, \mathrm{V}\right\rangle \quad \mathrm{d} \sigma
$$

$\underline{\text { Remarque } 4.2}$ - L'application de la proposition 3.2 nous donnerait le résultat suivant :

Proposition - Il existe un prolongement $\overline{\mathrm{z}}(\mathrm{T})$ de $\mathrm{z}(\mathrm{T})$ dans $\mathrm{H}^{1}(\mathrm{~B})$, G-dérivable par rapport à $T$ à valeurs dans $L^{2}(B)$ (dans $H^{k}(B)$ en se plaçant dans un contexte suffisamment régulier).

On montre aussi sans supplément de régularité que $\left.z(T)\right|_{\Omega_{0}}$ est dérivable à valeur dans $H^{1}\left(\Omega_{0}\right)$.

\section{Exemple $n^{\circ 2}$}

On suppose

$$
\stackrel{d a d \subset \mathrm{H}^{1}(\mathrm{~B}) ; \mathrm{z}_{\mathrm{d}} \in \mathrm{H}^{1}(\mathrm{~B})}{\mathrm{d}}
$$

On veut calculer le gradient de

$$
\mathrm{j}(\mathrm{T})=\frac{1}{2}\left\|\mathrm{z}-\mathrm{z}_{\mathrm{d}}\right\|_{\mathrm{L}}^{2}\left(\Omega_{\mathrm{T}}\right)
$$

avec

\footnotetext{
(1) $\chi_{\Omega_{0}}$ : fonction caractéristique de $\Omega$ 。
} 


$$
\left\{\begin{array}{l}
\Delta^{2} z+z=f \text { dans } \Omega T \\
\Delta z=0 \\
\frac{\partial}{\partial n} \Delta z=0
\end{array}\right\} \text { sur } \Omega_{T}
$$

On applique la proposition 3.1 à l'application

Introduisons l'êtat adjoint

$$
\begin{aligned}
& \overline{\bar{\sigma}}: \vartheta_{\mathrm{ad}} \times \mathrm{H}^{2}(\mathrm{~B}) \rightarrow\left[\mathrm{H}^{2}(\mathrm{~B})\right]^{\prime} \\
& {[\bar{\Phi}(\mathrm{T}, \varphi), \Psi]=\int_{\Omega \mathrm{T}}\{\Delta \varphi \Delta \Psi+\varphi \Psi-\mathrm{f} \Psi\} \mathrm{dv}}
\end{aligned}
$$

$$
\begin{array}{r}
\Delta^{2} p+p=z-z_{d} \\
\left.\begin{array}{rl}
\Delta p=0 & \text { dans } \Omega_{T} \\
\frac{\partial}{\partial n} \Delta p=0
\end{array}\right\} \quad \text { sur } \quad \Omega_{T}
\end{array}
$$

on obtient

$$
j^{\prime}(T, \theta)=\int_{\partial \Omega}\left\{\frac{1}{T}\left(z-z_{d}\right)^{2}-z p+f p\right\}<\vec{n}, V>d \sigma
$$

\section{Exemple ${ }^{\circ} 3$}

On suppose

$$
\begin{aligned}
& y_{\text {ad }} \subset d_{5} \\
& \leqslant H^{1}(B)
\end{aligned}
$$

l'équation d'état est la suivante

$$
\left\{\begin{array}{c}
\Delta^{2} z=f \\
z=0 \\
\frac{\partial z}{\partial n}=0
\end{array}\right\} \text { dans } \Omega_{T}
$$

la fonctionnelle est identique à celle de l'exemple 2 et l'on prend pour application $\bar{\Phi}$

$$
\begin{aligned}
& \because \theta_{\mathrm{ad}} \times \mathrm{H}^{4}(\mathrm{~B}) \rightarrow\left[\begin{array}{lll}
\mathrm{L}^{2}(\mathrm{~B}) \times \mathrm{H}^{1}(\mathrm{~B}) & \mathrm{H}^{1}(\mathrm{~B})
\end{array}\right] \\
& \forall\left(\mathrm{T}, \varphi, \Psi_{1}, \Psi_{2}, \Psi_{3},\right) \in \mathrm{Q}_{\mathrm{ad}} \times \mathrm{H}^{4}(\mathrm{~B}) \times \mathrm{L}^{2}\left(\mathrm{~B} \times\left[\mathrm{H}^{1}(\mathrm{~B})\right]^{2}\right. \\
& {\left[\bar{\Phi}(\mathrm{T}, \varphi),\left(\Psi_{1}, \psi_{2}, \psi_{3}\right)\right]=} \\
& \int_{\Omega T}^{5}(2 z-f) \Psi_{1} d v+\int_{\partial \Omega T}\left(z \Psi_{2}+\frac{\partial z}{\partial n} \Psi_{3}\right) d \sigma
\end{aligned}
$$

On en déduit l'équation adjointe

$$
\left\{\begin{array}{l}
\Delta^{2} p_{1}=z-z d \text { dans } \Omega_{T} \\
p_{1}=\frac{\partial p_{1}}{\partial n}=0 \\
p_{2}=\frac{\partial}{\partial n} \Delta p_{1} \\
p_{3}=-\Delta p_{1}
\end{array}\right\} \text { sur } \partial \Omega_{T}
$$

et le gradient

$$
j^{\prime}(T, \theta)=\int_{\partial \Omega_{T}}\left\{\frac{1}{2}\left(z-z_{d}\right)^{2}+\Delta z \Delta p\right\}<\vec{n}, v>d \sigma
$$


Cette mêthode $s^{\prime}$ applique aussi au contrôle de l'interface d'un système gouverné par un problème de transmission défini dans l'ouvert B tout entier (cf. [6] ) .

\section{CONCLUSION}

La méthode que nous avons présentée s'applique aux problèmes de contrôle par un domaine de systèmes régis par des problèmes bien posés suffisamment réguliers, linéaires ou non linéaires. Elle se généralise au cas de problèmes de valeurs propres; on retrouve alors les formules de Hadamard.

On peut aussi adapter cette mêthode à différents choix de paramétrage, et l'utilisation de ces formules dans des algorithmes du type gradient acceléré donne des rêsultats encourageants (cf [4] pour des résultats numériques) . 


\section{REFERENCES}

[1] J.CEA, A。 GIOAN, J.MICHEL

[2] G.CHAVENT, Thèse, Paris, 1970

[ 3 ] R.COURANT and D.HILBERT, Methods of Mathematical Physics, Vol I, Interscience, New York

[4] A.DERVIEUX, B.PALMERIO, Thèses de 3ème Cycle, Nice, 1974

[5] A.DERVIEUX, B.PALMERIO, Communication aux Journées sur le Contrồle, l'Identification ou la Localisation d'un domaine géométrique, Nice, Octobre 1974.

[6] A.DERVIEUX, B.PALMERIO, C.R.A.S. , 1975, t.280, pp.1697-1700 et $1761-1764$

[7] A.DERVIEUX, B.PALMERIO, B.ROUSSELET, à paraître

[ 8] P.GERMAIN, Mécanique des Milieux continus, Masson et Cie, Paris, 1962

[9] J.HADAMARD, Mémoire sur le problème d'analyse relatif à l'équilibre des plaques élastiques encastrées, oeuvre de Jacques Hadamard, C.N.R.S. , Paris, 1968

[10] J.L.LIONS, E.MAGENES, Problèmes aux limites non homogènes, Dunod, Paris, 1968

[11] F.MURAT, J.SIMON, Communication à ce congrès

[12] B.ROUSSELET, Communication à ce congrès

[13] L.SCHWARTZ, Cours d'Analyse, Hermann, Paris, 1967 . 